\title{
Influence of Donor Age on Splenomegaly in Chickens
}

Hisato Okabayashi and Ikuo Okada

Faculty of Fisheries and Animal Husbandry, Hiroshima University, Fukuyama, 720

The present paper was planned to investigate the influence of donor age on the expression of graft-versus-host (GVH) reaction in chickens.

The chickens used in the experiments were the $H$ and $L$ lines of White Leghorns, which have been selected for and against the donor competence of splenomegaly, respectively, and the $\mathrm{N}$ line was the fundamental population of the two lines. Seventeen- to 107-day old chickens of the three B blood group genotypes ( $A A,, A G$ and $G G$ ) in these lines were used as donors. The donor competence to elicit a GVH reaction was tested with the splenomegaly assay. Embryos of the $\mathrm{N}$ line which did not have the same B locus alleles as the donor were used as recipients. A dose of $0.05 \mathrm{ml}$ of blood was injected intravenously into each 14 -day old embryo and 5 days later the embryos were killed to determine the spleen weights. The degree of embryonic splenomegaly was used as a quantitative index of donor competence.

The relationship between the age and the splenic enlargement in each line is presented in Fig. 1. The mean spleen weights of embryos increased gradually until 106 days of donor age, though there was considerable fluctuation. During 60 to $7 \mathrm{C}$ days of age, the degree of splenomegaly stayed unusually low level, though the cause of decrease was unknown. There were observed differences between the lines. As expected, the donor competence of the H line was generally larger than that of the L line. Mean spleen weights fluctuated in parallel above that of the I line. On the other hand, in the $\mathrm{N}$ line, no consistent trend was observed. 


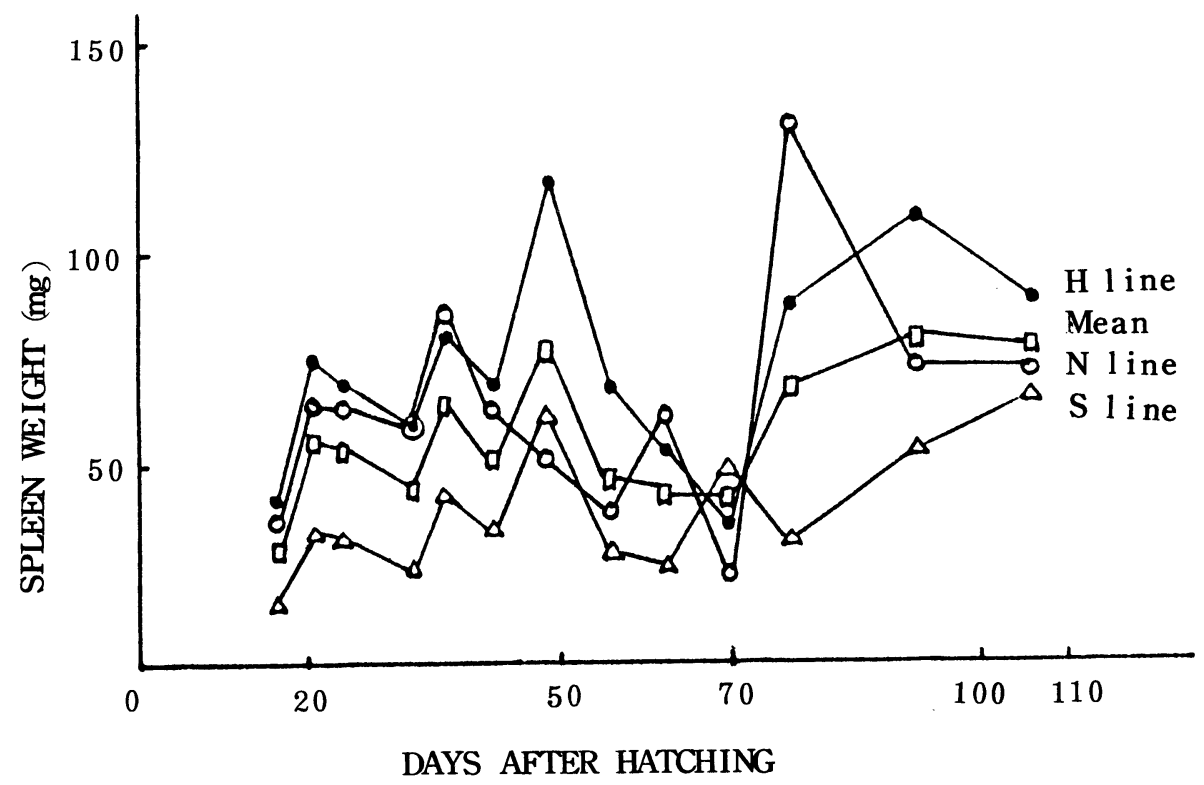

Fig. 1. The change of the splenic enlargement due to the donor age.

Table 1. Mean spleen weights of embryos inoculated with blood of 1- and 2year old chickens.

\begin{tabular}{ccc}
\hline Age & 1 & 2 \\
\hline $\begin{array}{l}\text { Mean spleen } \\
\text { weight (mg) }\end{array}$ & 109.8 & 72.6 \\
\hline
\end{tabular}

The difference is significant at 0.01 level.

Seto (1968) reported that the immunological competence of blood of White Ieghorns, as measured with GVH reaction, increased rapidly in the first 3 weeks, then at a slower rate, and reached a flateau level at about 12 weeks of age. The rresent results are generally consistent with his results, except for the decrease during 60 to $7 \mathrm{C}$ days of donor age.

Table 1 presents the comparison of donor competences of one and two 
year old chickens. One-year old donors induced larger spleen weights in embryos than did two-year old ones. The difference was statistically significant at 0.01 level. This suggests the decline of splenomegaly-inducing capacity after maturation.

\section{Reference}

Seto, F. 1968. Variations in the graft-versus-host reaction (GVHR) capacity of growing chickens. Transplantation, $6: 771-7 \varepsilon 2$. 